\title{
Economía circular: un camino hacia un Quito más sostenible
}

\section{Circular economy: a path towards a more sustainable Quito}

Flavio Roberto Arroyo Morocho

Dely Nathalia Bravo Donoso

Mario Augusto Rivera Valenzuela

Universidad Central del Ecuador, Ecuador

Autor para correspondencia: frarroyo@uce.edu.ec; dnbravo@uce.edu.ec; mariverav@uce.edu.ec Fecha de recepción: 18 de julio de 2018 Fecha de aceptación: 15 noviembre del 2018

Resumen: El presente artículo muestra la importancia de la Economía Circular en la actualidad y como esta se debe implementar en las empresas de la ciudad de Quito para construir un cambio radical en nuestro sistema económico y en nuestro medioambiente. Los últimos 150 años de evolución industrial han estado dominados por un modelo de producción y consumo lineal, en donde los productos/bienes son producidos a partir de materias primas, después son vendidos, utilizados y finalmente desechados como residuos. Frente a la actual economía global y el aumento de evidencias que apuntan a la creciente escasez y sobreexplotación de recursos, nace la necesidad de la búsqueda de un nuevo modelo económico (negocio). La economía circular presenta un cambio radical para lograr una economía sustentable para la sociedad, priorizando el cuidado y el uso de recursos, reduciendo el impacto ambiental que generamos. Finalmente se analiza la situación actual de la industria ecuatoriana las cuales buscan cambiar de un modelo lineal a un modelo circular, procurando minimizar riesgos, costos e impactos ambientales.

Palabras claves: economía circular; reciclaje; sostenible; impactos ambientales; economía lineal; industria ecuatoriana; modelos de negocio; quito

Abstract: This article shows the importance of Circular Economy today and how it should be implemented in companies in the city of Quito to build a radical change in our economic system and our environment. The last 150 years of industrial evolution have been dominated by a linear production and consumption model, where products / goods are produced from raw materials, then sold, used and finally disposed of as waste. Faced with the current global economy and the increase in evidence pointing to the growing scarcity and overexploitation of resources, the need arose to search for a new economic model (business). The circular economy presents a radical change to achieve a sustainable economy for society, prioritizing the care and use of resources, reducing the environmental impact that we generate. Finally, the current situation of the Ecuadorian industry is analyzed, which seeks to change from a linear model to a circular model, trying to minimize risks, costs and environmental impacts.

Key words: circular economy; recycling; sustainable; environmental impacts; economy linear; ecuadorian industry; business models; quito 


\section{Introducción}

En la actualidad la mayor parte de las empresas ecuatorianas y empresas extranjeras que operan en el país siguen el modelo económico lineal, que no ha cambiado desde la primera revolución industrial, el cual consiste en la extracción de materia prima, la producción de bienes, el consumo y la generación de residuos.

En la segunda mitad del siglo XVIII la revolución industrial cambió radicalmente las formas de producción y consumo, fomentando una rápida transformación de los otros descentralizados, artesanales y lentos sistemas de producción. Ello fue posible debido a diversos factores, como el desarrollo tecnológico (máquina de vapor), el desarrollo organizacional, la globalización de mercados y recursos (colonialismo), la disponibilidad de energía asequible (carbón) y la mano de obra; esto último incrementando las dinámicas de urbanización. (Jordi Morató, 2017)

La humanidad se enfrenta a una crisis ecológica de gran magnitud. Problemas como el agujero en la capa de ozono, el incremento de gases de efecto invernadero, la pérdida de biodiversidad, el agotamiento de recursos tanto renovables como no renovables, la contaminación del suelo y del aire, entre otros, lo denotan. Tanto las administraciones como las instituciones científicas aceptan este hecho como incontrovertible. Los causantes de estos fenómenos lo son en grado diferente, y desigual es también el grado en que los afectados sufren sus consecuencias. Las emisiones de clorofluoro-carburo, por ejemplo, se produjeron esencialmente en los países industrializados y sus efectos más severos se perciben y percibirán sobre todo en las zonas más cercanas a los polos; la cantidad de emisiones de gases de efecto invernadero correlaciona en gran medida con el nivel de renta de los países, que no tienen por qué ser los más afectados por los cambios drásticos en el clima ni por la subida del nivel del mar, etc. (Medioambiente, 2016)

Cien millones de personas viven en áreas susceptibles a la contaminación del aire. Uno de cada cuatro tramos fluviales está clasificado como severamente contaminado, en especial por aguas domésticas y por la producción agrícola, industrial y de exportación. Tres millones de kilómetros cuadrados de suelo han sido afectados por degradación generada por el hombre. (Paucar, 2017) 


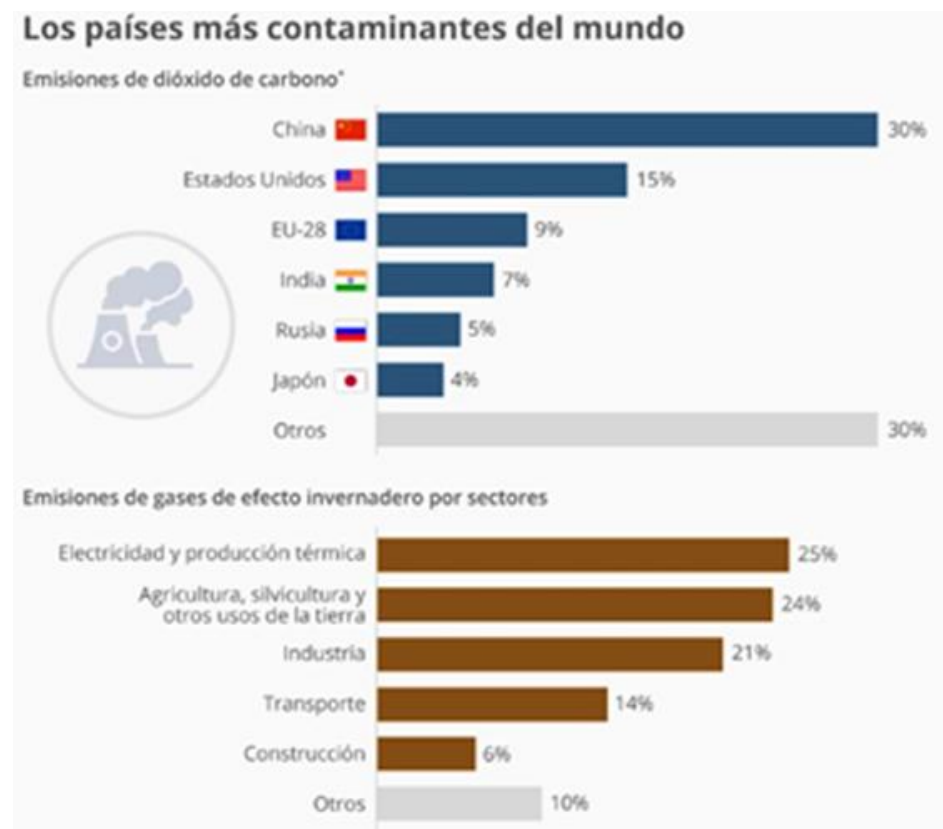

Figura 1. Los países más contaminantes del mundo y las emisiones por sectores.

Fuente (Moreno, 2017)

En Quito se produce alrededor de 2.000 toneladas diarias de basura de las cuales, el 55\% son desechos orgánicos, es decir, desperdicios de verduras, frutas, comestibles, etc.

(CONQUITO, 2017). La Economía Circular supone un cambio radical de los sistemas de producción y consumo actuales. El cambio se debe dar hacia sistemas que sean regenerativos a partir de su diseño, para mantener el valor de los recursos (materiales, agua, suelo y energía) y de los productos limitando exponencialmente, los insumos de materias primas y energía. Esto evitará la creación de residuos e impactos negativos derivados, mitigando las externalidades negativas para el medioambiente, el clima y la salud humana. (Jordi Morató, 2017)

A lo largo de su evolución y diversificación, nuestra economía industrial nunca se ha movido más allá de una característica fundamental establecido en los primeros días de industrialización: un modelo lineal de recurso - consumo. Empresas extraen materiales, aplican energía y trabajo para fabricar un producto y venderlo hasta el consumidor final quien luego lo descarta cuando no ya cumple su propósito. Mientras grandes avances se han hecho en la mejora de la eficiencia de los recursos, cualquier sistema basado en el consumo en lugar de en el uso restaurador de recursos implica pérdidas significativas todo el tiempo en la cadena de valor. (MacArthur, 2014). Los modelos comerciales de economía circular se dividen en dos grupos: aquellos que fomentan la reutilización y extienden la vida útil a través de la reparación, refabricación, actualizaciones y modernizaciones; y aquellos que convierten los productos viejos en recursos nuevos reciclando los materiales. Las personas, de todas las edades y habilidades, son fundamentales para el modelo. La propiedad da paso a la mayordomía; los consumidores se convierten en usuarios y creadores. La refabricación y reparación de productos viejos, edificios e infraestructura crea trabajos calificados en talleres locales. Las experiencias de los trabajadores del pasado son instrumentales. (Stahel, 2016). 


\section{CLOSING LOOPS}

Using resources for the longest time possible could cut some nations' emissions by up to $70 \%$, increase their workforces by $4 \%$ and greatly lessen waste.

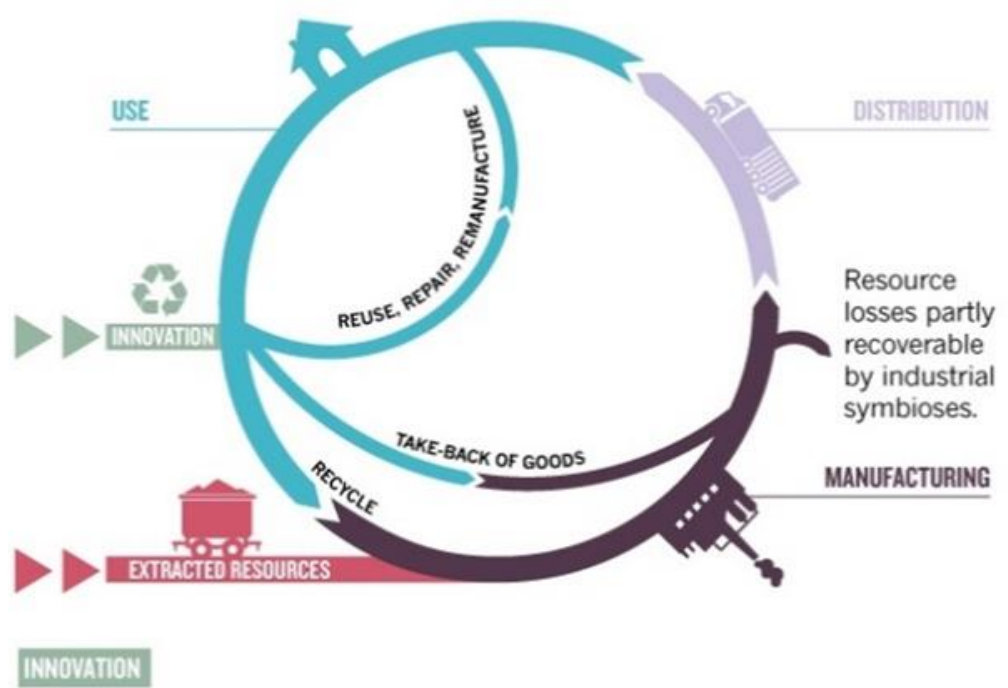

Research is needed to transform used goods into 'as-new' and to recycle atoms. EXTRACTED RESOURCES

Water, energy and natural resources enter the manufacturing process.

\section{MANUFACTURING}

Renewing used products lessens the need to make originals from scratch.

DISTRIIIION

Ownership transfers from manufacturer to consumer at point of sale.

USE

Is controlled by buyer-owner-consumers of goods, or by fleet managers who retain ownership and sell goods as services.

Figura 2. Bucles de Cierre: Uso de recursos

Fuente: (Stahel, 2016)

De esta manera este modelo económico busca independizarse del uso de materias primas no renovables incorporando nuevas tecnologías y aplicando modelos de negocios basados en la durabilidad, renovación, reutilización, reparación, re fabricación, capacidad de compartir y desmaterialización, creando un modelo circular que brinda oportunidades de negocio con una rentabilidad a gran escala. En sí las empresas que innovan en la aplicación de este modelo, pueden conseguir grandes utilidades, y generar ventajas competitivas en el mercado.

Recientemente, muchas empresas también han empezado a darse cuenta de que el sistema lineal aumenta su exposición a los riesgos, sobretodo el aumento del precio de los recursos y las interrupciones en su suministro. Cada vez más empresas se sienten constreñidas, por las alzas en los precios y el aumento en su volatilidad de un lado y por la elevada competitividad y el estancamiento de la demanda en determinados sectores por el otro. El fin del milenio marcó el punto de inflexión en el cual los precios reales de recursos naturales comenzaron a ascender, esencialmente borrando la reducción de precios reales obtenida a lo largo del siglo. (Sandoval, 2017) 


\section{Metodología}

\section{Economía Circular}

El profesional actual, la política y el desarrollo empresarial hablan sobre el concepto de Economía Circular (EC) formulado en el reino que se muestra en la figura 3.

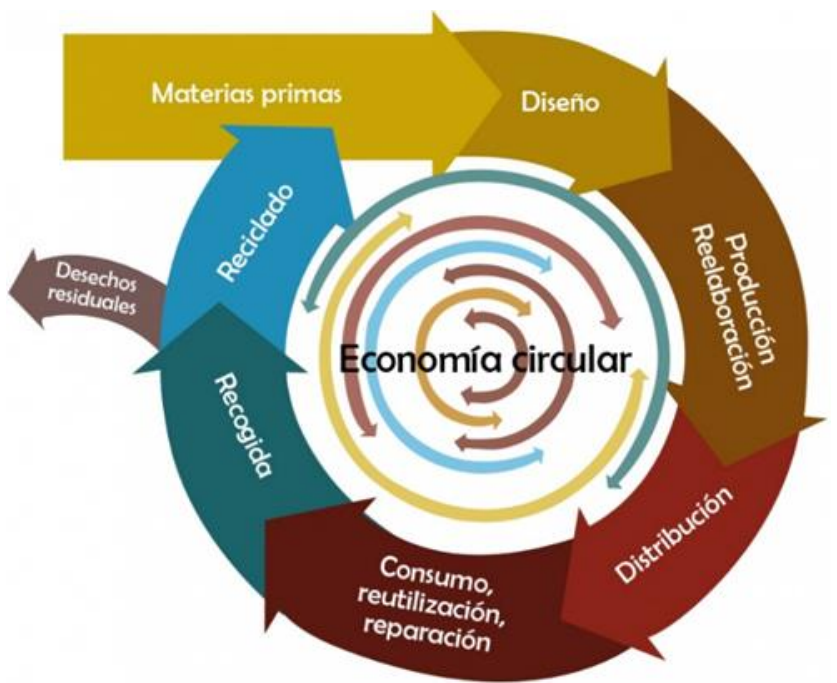

Figura 3. Representación integral de la lógica económica y empresarial incrustada en el concepto EC. Fuente: (Revertía, 2016).

Los círculos internos, reutilización del producto, re-fabricación y reacondicionamiento, demandan menos recursos y energía y son más económicos que el reciclaje convencional de materiales como materias primas de baja calidad. El tiempo del valor en los recursos gastados / vidas dentro de los círculos internos deben ser maximizado. Los materiales deben ser recuperados primero para su reutilización, reacondicionamiento y reparación, luego remanufacturado y solo más tarde para la utilización de la materia prima. De esta manera, la cadena de valor del producto y ciclo de vida conservan el mayor valor posible y calidad el mayor tiempo posible (Jouni Korhonen, 2017).

Una economía circular es un sistema industrial que es restaurativo o regenerativo por intención y diseño. Sustituye al concepto de fin de vida con restauración, cambios hacia el uso de energías renovables, elimina el uso de productos con químicos tóxicos, que perjudican la reutilización y reintegración a la biosfera, y tiene como objetivo la eliminación de desechos a través del diseño superior de materiales, productos, sistemas y modelos de negocios. (Bocken, Pauw, Bakker, \& Grinten, 2016)

La Economía Circular se basa en principios como la diversidad, la resiliencia y el pensamiento sistémico, que requieren un enfoque metabólico, integrando ciclos de materiales biológicos y tecnológicos. (Jordi Morató, 2017). La iniciativa circular exitosa reducirá la dependencia de recursos naturales, creando un valor para las empresas y los grupos de intereses. (Economy, 2017). Para lograr implementar este modelo económico circular debemos conocer sus tipos de negocio en los cuales puede estar presente, (Sandoval, 2017) explica 5 tipos de negocio que la economía circular permite que funcionen. 
Extraer: En el marco de la EC, el término "extraer" se refiere a la forma en que las industrias toman recursos del entorno, por tanto, las empresas deben intentar hacer un uso más eficaz y responsable de los recursos biológicos y técnicos. Esto implica que las empresas pueden seleccionar los proveedores y los materiales que utilizan, de acuerdo con criterios medioambientales que disminuyan su impacto en la naturaleza.

Transformar: Tan pronto se obtienen los recursos, se debe procurar el desarrollo de las mejores prácticas tecnológicas e innovaciones ecológicas (eco-innovaciones) para que tanto el producto o servicio como su proceso se realicen de la manera más sostenible posible.

Distribuir: Esta fase tiene que ver con la forma en la que el producto o servicio se entrega al cliente. Las empresas deben garantizar la trazabilidad de sus productos y de manera eficiente reducir el impacto ambiental, tanto en rutas, como embalajes, como, a través de distintas prácticas, como la logística inversa.

Usar: En cuanto el producto está a disposición de los consumidores u otras empresas, la EC propone reducir el impacto de la energía asociada al uso del producto o la eficiencia del propio producto. La eficiencia del producto o servicio puede ser mejorada a través de la reutilización como producto de segunda mano o la reparación.

Recuperar: Por último, en la EC, los residuos pueden ser recuperados de dos maneras: como un recurso biológico que puede ser devuelto a la biosfera o como un recurso técnico que puede ser reincorporado a un proceso industrial.
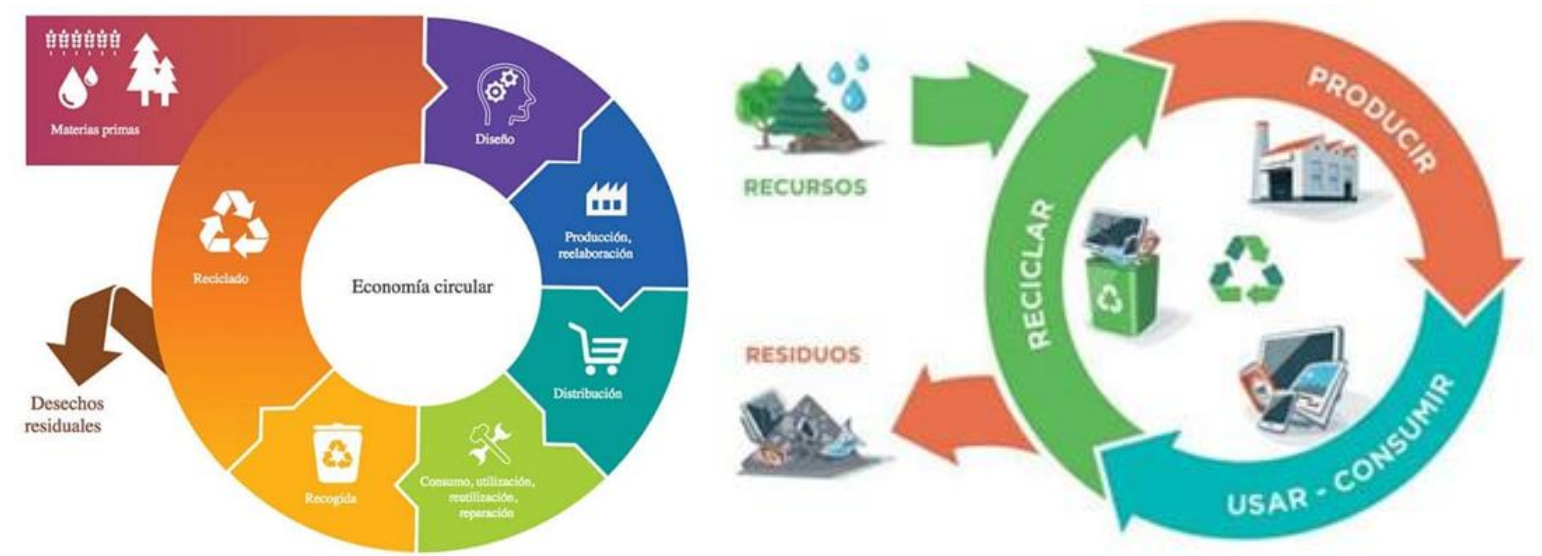

Figura 4. Representación del modelo de negocios de la Economía Circular. Fuente: (Ovacen, s/f)

\section{Oportunidad de Innovación con modelo económico circular.}

En el proceso de construcción de los nuevos sistemas de indicadores de Economía Circular hay que tener en cuenta que una parte considerable de éstos aún no están totalmente desarrollados, especialmente los relativos a la prevención del uso excesivo de materias primas, el ecodiseño y la ecoinnovación. En cambio, se ha avanzado notablemente en el uso eficiente de materiales y la gestión de residuos, aunque se reconoce de antemano que son necesarios mayores esfuerzos para dar una visión más completa y detallada de los progresos de la Economía Circular hacia el desarrollo sostenible. (Jordi Morató, 2017). 
Agua en caja es una opción sostenible y mucho más eficiente que una botella de plástico, porque gracias a que está hecha del $75 \%$ de materiales renovables, no genera tantos residuos y además proporciona un envase más fácil de reciclar. Esta propuesta deriva de que el plástico no es una buena opción, ya que contamina nuestro planeta, además de dañar mares, océanos y la supervivencia de los animales marítimos, entre otros. (Patricia, 2018). Canon maximiza el valor de su capital fabricado al recolectar equipos usados en el mercado, reutilizan al menos el $80 \%$ de los materiales. También reduce las emisiones de gases de efecto invernadero asociadas con la materia prima, las piezas y la fabricación. (Economy, 2017).

La nueva tecnología jugará un papel importante en el movimiento del entorno construido hacia la economía circular. Con la evolución de nueva tecnología que incluye plataformas digitales, pasaportes de productos, impresoras 3D y sensores de etiquetado, está claro que la innovación futura alterará el proceso de construcción del ciclo de vida. (Carra \& Magdani, 2016).

\section{Estrategias de diseño de productos circulares}

Integrando preocupaciones de economía circular en una etapa temprana en el proceso de diseño del producto es importante, porque una vez las especificaciones del producto están siendo hechas, solo pequeños cambios son usualmente posibles es difícil hacer cambios, una vez recursos, infraestructuras y actividades se han comprometido con un determinado diseño de producto. (Bocken, Pauw, Bakker, \& Grinten, 2016). Diseñar productos de larga duración es la primera estrategia de diseño. Se trata de asegurar un largo período de utilización de los productos. Dentro esta categorización, "Diseñar para el apego y la confianza" se refiere a la creación de productos que serán amados, queridos o confiado más tiempo.

"Diseño para la durabilidad" se relaciona con el físico durabilidad, por ejemplo, el desarrollo de productos que puede desgastarse sin romperse. Material la selección de durabilidad es una parte importante del diseño proceso. "Diseño para confiabilidad" se refiere a diseñar para alta probabilidad de que un producto opere a través de un período especificado sin experimentar una falla de carga, cuando se mantiene de acuerdo con las especificaciones del fabricante instrucciones. (Bocken, Pauw, Bakker, \& Grinten, 2016)

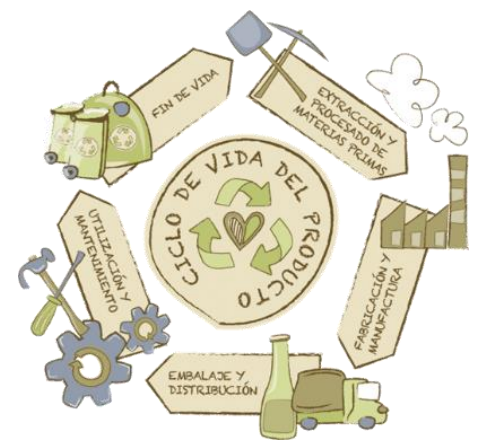

Figura 5. Ciclo de vida de un producto.

Fuente: (Canga Cabañes, 2015) 
La segunda estrategia de diseño para ralentizar los bucles de recursos es "Diseño para la extensión de la vida del producto. " Esta estrategia se refiere con la extensión del período de uso de los bienes a través de la introducción de bucles de servicio para extender la vida útil del producto, incluida la reutilización del producto en sí, mantenimiento, reparación, y actualización técnica, y una combinación de estos. (Bocken, Pauw, Bakker, \& Grinten, 2016)

\section{Enfoque Metodología Circularity Canvas}

Esta metodología te ayuda a dar el primer paso hacia la circularidad y a concebir modelos de negocio económica, social y ambientalmente sostenibles. Su objetivo es reducir la generación de residuos integrando los principios de Economía Circular y Cradle to Cradle, desde las etapas más tempranas de la concepción de un modelo de negocio.

La Metodología Circularity Canvas está basada en la herramienta Business Model Canvas (BMC), particularizando sus bloques básicos hacia modelos de negocio circulares. Se plantea como un camino circular, comenzando con un BMC inicial orientado a la Economía Circular y finalizando con su ajuste tras la identificación de propuestas de valor entre los diferentes grupos de interés involucrados en el marco completo de un Modelo de Negocio Circular.

Interés nacional en cambio de modelo lineal.

La transición a un modelo de economía circular es ahora una prioridad en las políticas de la UE y la innovación es un elemento clave en este proceso. Actualmente en el Ecuador, el 80\% de empresas no invierten en protección ni reducción del impacto ambiental y solo el $2 \%$ han incluido sistemas de gestión ambiental. Datos revelados por el Instituto Nacional de Estadísticas y Censos (INEC).

Este análisis realizado a más de 3572 empresas, dedicadas a la explotación minera y canteras, industrias manufactureras, comercio al por mayor y menor, hoteles, restaurantes y servicios, determinó que en la región Amazónica es donde existe mayor cantidad de estudios de impacto ambiental ( 2 de cada 10 establecimientos), mientras que en la Sierra se encuentra el mayor porcentaje de establecimientos con licencia ambiental (2 de cada 10).

Es fundamental resaltar la importancia que tiene el diseño de los productos (bienes y servicios) para que el flujo de materiales y energía realmente sea circular. Es por esto que múltiples investigaciones se han dedicado a buscar el conjunto de parámetros y estrategias que pueden guiar a las empresas en el diseño de productos eco-innovadores. Este tipo de productos sostenibles pueden permitirles a las organizaciones generar una ventaja competitiva en mercados locales e internacionales, incrementar la percepción de calidad percibida por parte de sus clientes, e impulsar el desarrollo de ciclos de eco-innovación en las empresas.

Alrededor de 2.000 toneladas al día, de residuos domésticos e industriales no peligrosos, se producen en Quito y van directamente al Relleno Sanitario "El Inga". "Quito a Reciclar" es un proyecto destinado a la Gestión Integral de Residuos, la cual busca la participación de la ciudadanía para el cuidado del medio ambiente, este proyecto permite que cerca de 200 familias, de ex mineros, hoy sean gestores ambientales y que actualmente sean los encargados de la recolección diaria de residuos reciclables; contando así con un sueldo fijo y una práctica fuera de 
peligro. (Secretaria de Ambiente Alcaldía, 2015). En el año 2015, el DMQ produjo un promedio de 2.037 toneladas al día de residuos domésticos e industriales no peligrosos en una población urbana y rural de 2`551.721 habitantes. La producción de residuos recolectada per cápita fue de 0,842 kg/día/hab. (Secretaria del Medio Ambiente Quito, 2015)

La economía circular se enfoca en el crecimiento económico que está en línea con el desarrollo ambiental y económico sostenible. Es una economía construida a partir de sistemas de producción y consumo de la sociedad, maximizando el servicio producido a partir del material y la energía, el éxito de la economía circular termina contribuyendo a tres dimensiones del desarrollo sostenible. (Korhonen, Honkasalo, \& Seppälä, 2017)

La CE apoyada en los fundamentos de la escuela ecologista, la cual propone un cambio al paradigma "reducir, reutilizar y reciclar" por una transformación más profunda y duradera, la cual permita disminuir el impacto que causan las actividades humanas sobre el medio ambiente. (Lett, 2014)

También se dirige hacia un nuevo paradigma, lo cual implica una nueva modalidad de hacer productos desde su origen, desde el diseño, y permitan hacer negocios relacionados con el crecimiento económico de la sociedad, la sustentabilidad ambiental y la disminución de riesgos por la volatilidad y la incertidumbre del precio de la materia prima y los recursos energéticos. (Lett, 2014)

\section{Resultados}

Si bien el continente europeo es pionero en implantar este modelo económico en su industria, Latinoamérica ya motiva e impulsa el desarrollo de la economía circular en su industria. El CAF -banco de desarrollo de América Latina apoyó el primer Foro de Economía Circular de América Latina, en el cual se reunió durante dos jornadas a representantes locales e internacionales, con el fin de debatir y aprender las formas de dinamizar a los actores claves de la sociedad e impulsar la transición de la actual economía lineal hacia un modelo circular, que proponga un cambio sistémico radical.

Verónica Arias, Secretaria de Ambiente del Distrito Metropolitano de Quito, manifiesta que sueña y cree que es posible una ciudad ambientalmente responsable. En el análisis que realiza determina que es fundamental que su crecimiento esté asociado al límite natural y racional de sus ecosistemas, que su territorio se piense y planifique tomando en cuenta sus activos ambientales, a la par que las decisiones consideren minimizar los impactos negativos que generan contaminación. (Arias, 2017). En el parque la carolina se generan 7 toneladas de residuos diarios, siendo que al mes se registran un promedio de 220 mil visitantes que asisten al parque. El 4 de febrero de 2018, EMASEO recolecto 65,5 toneladas de basura en 296 recintos electorales del DMQ. 2300 toneladas de basura en Quito, tras fiestas de fin de año, 60 mil toneladas de basura en navidad. (EMASEO, 2017).

Tabla 1. Toneladas de residuos según el sector y época

\begin{tabular}{lll}
\hline Residuos & Toneladas & Año \\
\hline
\end{tabular}




\begin{tabular}{lll}
\hline Diarios & 2000 & 2017 \\
Parque la Carolina & $7 /$ día & 2018 \\
$\begin{array}{l}\text { Recintos electorales } \\
\text { 2018 }\end{array}$ & 65,5 & 2018 \\
Fin de Año & 2300 & 2017 \\
Navidad & $\mathbf{6 0 0 0 0}$ & $\mathbf{2 0 1 7}$ \\
\hline
\end{tabular}

Fuente: EMASEO

En el Ecuador existen un total de 704.556 empresas, este fue uno de los resultados que arrojó el Directorio de Empresas Nacional, elaborado por el Instituto Nacional de Estadística y Censos. Algunos datos revelan que el 19\% de las empresas están en Quito, siendo la ciudad con mayor número de empresas, seguida por Guayaquil.

Tabla 2. Top 10 de las empresas en el Ecuador:

\begin{tabular}{ll}
\hline Empresas & Ingresos anuales \\
\hline Corporación Favorita & $1.989,01$ \\
Claro & $1.540,23$ \\
Banco Pichincha & $1.132,45$ \\
Corporación El Rosado & $1.051,62$ \\
Pronaca & 995,82 \\
Gm Obb & 908,28 \\
Dinadec & $1.051,62$ \\
Telefónica Movistar & 680,47 \\
Grupo Difare & 641,14 \\
Tía & $\mathbf{6 0 2 , 8 0}$ \\
\hline
\end{tabular}

Fuente: Revista Ekos 2017

La corporación favorita desde el año 2008 incorporó el uso de fundas biodegrables y reutilizables en su cadena de supermercados, actualmente impulsa el uso de fundas biodegrables y da consejos en su página web sobre el uso que se puede dar como: sembrar plantas, reutilizarlas, o enterrarlas para el proceso de biodegradación. Además, promueve el uso de bolsas reutilizables en varios aspectos como: Cartucheras, material para manualidades, mochilas, etc.

El Ecuador utiliza alrededor de 257 millones de fundas plásticas por año, lo que repercute negativamente al medio ambiente. Según los datos del INEC en el año 2016 el consumo de bolsas reutilizables es del $18.9 \%$. Desde este año el ministerio de medio ambiente ha impulsado el uso de estos productos. La empresa Claro ocupa el segundo lugar en el ranking sin embargo no incorpora nuevas tecnologías para disminuir su impacto en el medioambiente.

La humanidad genera 1 billón de toneladas de residuos anualmente. Y la basura va en aumento. La que más preocupa es quizás la electrónica (e-waste): unos 42 millones de toneladas al año, de las que solo se destinan al reciclaje un $17 \%$. Si se recuperarán todos los materiales del e-waste, se obtendrían 52.000 millones, y, lo que es más importante, se evitarían la 
contaminación del medio ambiente y los riesgos para la salud. La economía circular permite abordar este problema.

Quito emite cada año 5,1 millones de gases de efecto invernadero a la atmósfera. En esta cantidad se contemplan todas las emisiones que son generadas por el transporte, la industria, los residuos sólidos y las actividades comerciales y domésticas, cada año.

Es la primera vez que se conoce este valor con cifras concretas. De este total, 2,8 millones de toneladas de dióxido de carbono son producidas por el transporte público y particular.

La Industria Manufacturera es parte fundamental de la economía en el país, al tratar el término industria hacemos referencia al proceso mediante el cual se transforma la materia prima en un producto para su uso final o que servirá de insumos para otros procesos productivos. (Ekos, 2015)

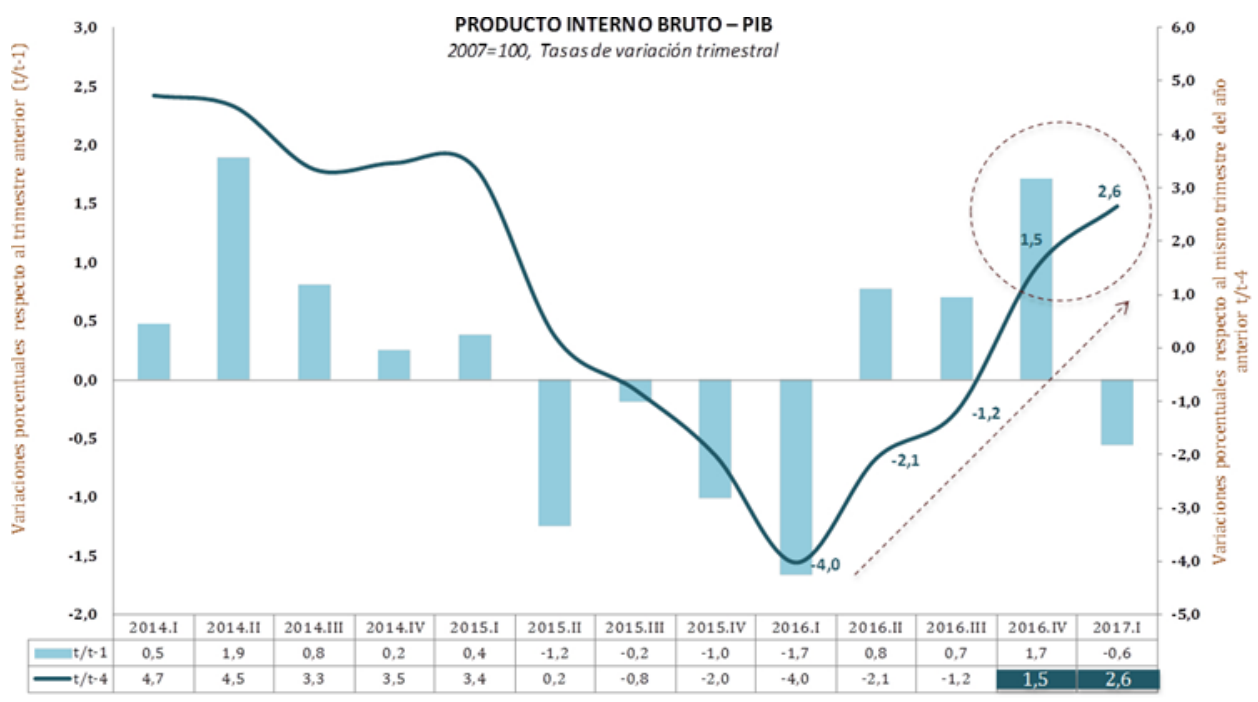

Figura 6. Evolución del PIB y tasa de variación anual.

Fuente: (Banco Central del Ecuador, 2017)

En lo que a composición se refiere, la elaboración de alimentos y bebidas es la industria más importante en el país con un peso de $38 \%$ dentro del total del producto manufacturero y un producto generado de USD 5.297 millones. A éste le sigue la industria química con un peso de $11 \%$, siendo una actividad considerada prioritaria en las políticas gubernamentales. En ercer lugar, se encuentran los productos minerales no metálicos $(9 \%)$ seguidos por la industria textil y de cuero (7\%) y metálica (7\%). (Ekos, 2015) 

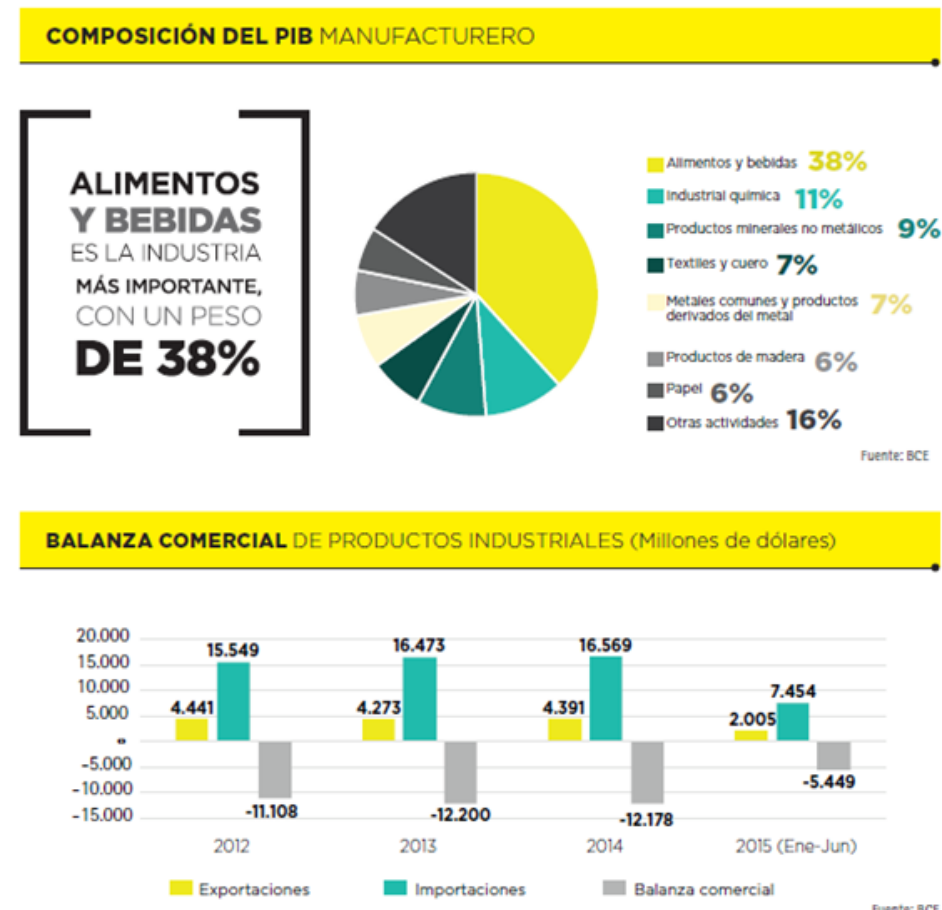

Figura 7. Composición manufacturera en el Ecuador.

Fuente: (Ekos, 2015)

Telefónica (Movistar) se está digitalizando la gestión de residuos tecnológicos en Europa y Latinoamérica, con ayuda de una herramienta, denominada GreTel (Gestión de Residuos de Telefónica) y desarrollada por la start-up gallega Teimas Desenvolvemento. La nueva plataforma ya funciona en España y Colombia y este año llegará a los otros 15 países en los que Telefónica tiene presencia (Tejedor, 2017)

GreTel una herramienta para un modelo de negocio circular, busca reducir aún más su impacto ambiental mediante planes de minimización de generación de residuos, reciclaje y reúso interno de, principalmente, aparatos eléctricos y electrónicos. Consiste en el diseño e implementación de un Sistema de Gestión de Residuos, tipo web, cuyo objetivo principal es el de aumentar la valorización de los residuos gestionados mediante la digitalización del proceso, así como la minimización de los riesgos asociados, englobando y poniendo en práctica aspectos importantes como: la economía circular, uniformidad, sencillez, visión global, adaptabilidad, consistencia, gestión simplificada, accesibilidad y seguridad (Tejedor, 2017)

\section{Actualidad Distrito Metropolitano (DM) de Quito: Cantidad de residuos generados en el DM de Quito}

Una vez realizado el levantamiento de catastro para el Manejo de Residuos de las Industrias en el Distrito Metropolitano de Quito con la ayuda de la Empresa Pública Metropolitana de Gestión Integral de Residuos Sólidos “EMGIRS EP”. El objetivo de esta actividad es obtener información sobre los residuos industriales no peligrosos, para emprender nuevos procesos más amigables con el ambiente. 
EMGIRS EP, visita a diferentes industrias de la capital a fin de realizar aproximadamente 1.800 registros, en 30 días. En la actualidad, en 12 industrias de Quito, se produce un promedio de 1.500 toneladas de residuos industriales no peligrosos. En el levantamiento del catastro se identificarán cuántas industrias existen, el tipo y la cantidad de desechos que generan. Los residuos industriales no peligrosos son: el aceite, lodos industriales, desechos de papel, lanas, telas, fragmentos de cerámicas, madera, tejas, llantas, entre otros (EMGIRS -EP, 2016).

El sector empresarial relacionado con la producción e importación de neumáticos se propone recuperar alrededor de 750 mil unidades fuera de uso, que serían procesadas por la industria de reciclaje y las plantas de reencauche que funcionan en el Ecuador, con lo cual se prevén minimizar los impactos medio ambientales que existen actualmente en el país.

Dentro del Plan Maestro de Gestión Integral de residuos del Distrito Metropolitano de Quito existen varios objetivos que se pueden vincular con modelos de negocios de economía circular, a continuación, presentamos algunos objetivos en cuales se puede evidenciar el apoyo e interés del municipio de Quito en construir modelos de negocios que intervengan en la gestión integral de residuos de la ciudad.

La secretaria de ambiente plantea varios proyectos de cuidado ambiental, en los cuales existen grandes oportunidades de negocios un ejemplo de ello es "Quito a Reciclar" es una campaña destinada a la Gestión Integral de Residuos Sólidos que busca la participación de toda la ciudadanía para el cuidado del ambiente. Este proyecto impulsado por la Secretaría de Ambiente del Distrito Metropolitano de Quito, permite que cerca de 200 familias, de ex mineros, hoy sean gestores ambientales y que actualmente sean los encargados de la recolección diaria de residuos reciclables; contando así con un sueldo fijo y una práctica fuera de peligro (Secretaría de Ambiente Quito, 2017). Con este proyecto se pueden implementar varios negocios que se dediquen a la recolección de residuos reciclables y a partir de ello dar tratamiento para la reutilización como materia prima en la industria.

Actualmente existen 811 Puntos Limpios instalados tanto en instituciones públicas como en privadas, 12 barrios beneficiados con programas de recolección no mecanizada diferenciada a pie de vereda cuyo objetivo principal es involucrar a la comunidad a separar sus residuos sólidos desde la fuente, empoderando a los gestores ambientales de menor escala de la zona a recuperar el material reciclable bajo la modalidad a pie de vereda. (Secretaría de Ambiente Quito, 2017)

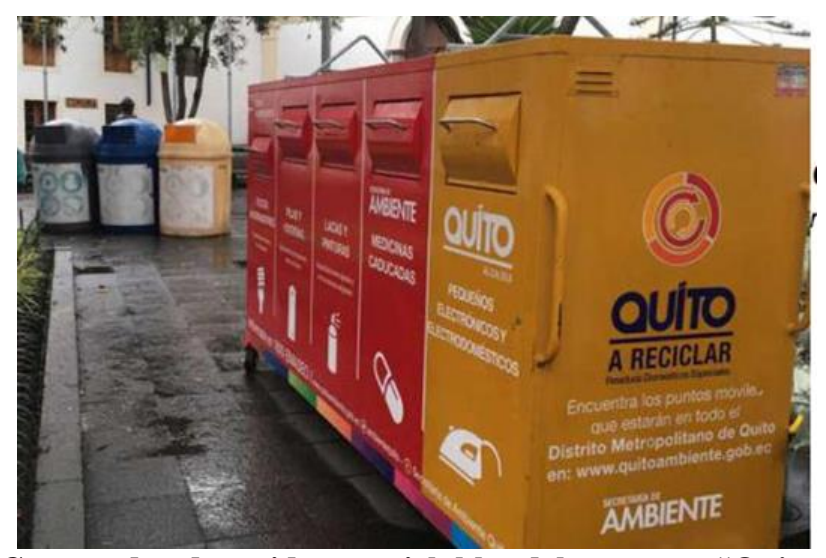

Figura 8. Contenedor de residuos reciclables del proyecto "Quito a reciclar". 
Fuente: (El Comercio, 2018)

Las oportunidades de negocio implementando el modelo de economía circular con un enfoque en el uso de energías renovables también es punto importante en el cual se puede trabajar en distintos de planes de tratamiento/ aprovechamiento de residuos planteados por la secretaría de ambiente.

Planta de generación eléctrica de captación de biogás: Las emisiones de biogás son otro subproducto de la descomposición de la materia orgánica en el relleno sanitario. El sistema de captación de biogás producido en el relleno sanitario se realiza mediante la colocación de pozos perforados en los que se inserta tuberías de polietileno de alta densidad que captan el biogás producido con un porcentaje de metano de 46\%, 16\% CO2 y 38\% de otros gases de efecto invernadero. La contaminación del metano es 24 veces más alta que el CO2 y puede ser aprovechada. Por tal razón, EMGIRS-EP instaló dos generadores con de 1MW cada uno, para el aprovechamiento energético del metano (biogás) captado. La planta de aprovechamiento energético comenzó su fase de pruebas de producción eléctrica en enero del 2016. Durante este mes, los dos motores pasaron por diferentes pruebas y calibraciones hasta llegar a una producción y funcionamiento óptimos. (Secretaría de Ambiente Quito, 2017)

Escombreras: Hoy en día, la ciudad Quito entrega 3.115 m3/día de escombros a las escombreras municipales. En la actualidad el Municipio opera dos escombreras, Troje 4 y Piedras Negras, con nuevos modelos que permite realizar un manejo técnico de la disposición final de los residuos sólidos urbanos, evita generar peligro para la salud o la seguridad pública y cuida el ambiente durante la operación y posterior a su clausura. El nuevo modelo de gestión de la escombrera contempla estacionamientos para vehículos, señalización, guardianía y controles, comedor, sistemas de comunicación y seguridad, baterías sanitarias, luz eléctrica, entre otros servicios. (Secretaría de Ambiente Quito, 2017)

La gestión local de cambio climático es uno de los ejes de la visión "Quito: Ciudad Sostenible", que propone un conjunto de políticas y acciones para responder frente a los impactos de este fenómeno global, así como para reducir las emisiones de gases de efecto invernadero (Reducción de Huella de Carbono Local), con el conjunto de medidas programadas para los sectores: movilidad, energía, residuos y bosques. (Secretaría de Ambiente Quito, 2017)

La Huella de Carbono calculada para la ciudad es en total 5.164.946 ton CO2eq, distribuida de la siguiente manera: sector transporte con el porcentaje más alto respecto al total de las emisiones (56\%) emite 2.902.402 ton CO2eq, en segundo lugar el sector residencial, comercial e institucional con una generación de emisiones de 1.016 .305 ton CO2e (20\%), el sector de residuos sólidos con 661.689 ton CO2e (13\%) y finalmente con un porcentaje de $11 \%$ el sector industrial con 584.550 ton CO2eq. (Secretaría de Ambiente Quito, 2017) 


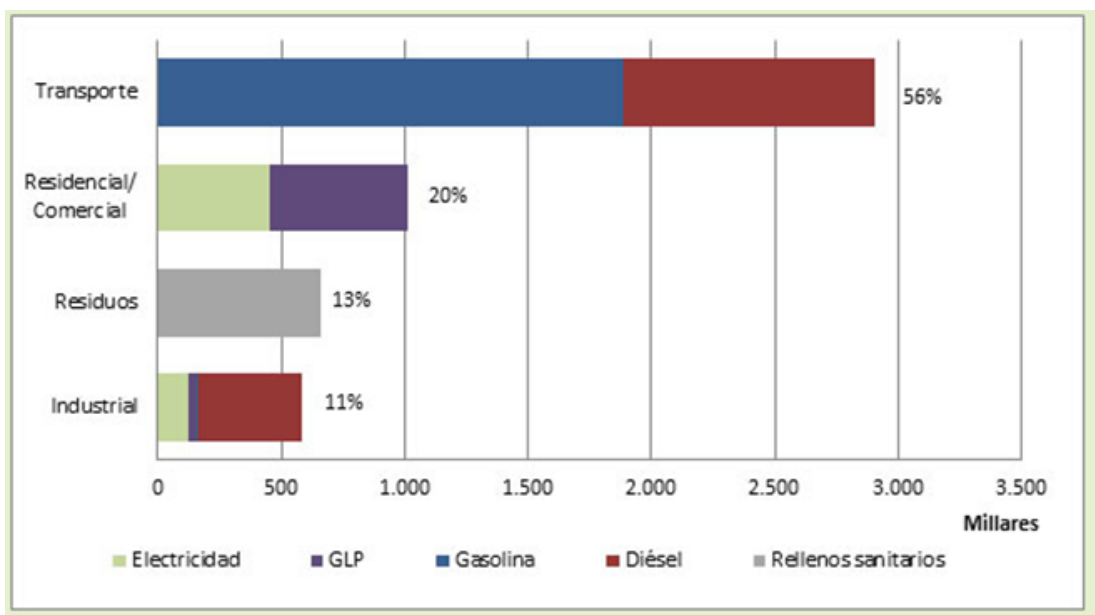

Figura 9. Huella de Carbono total según sector y fuente de emisión (en toneladas CO2e). Fuente: (Secretaría de ambiente Quito, s/f)

Para esto se diseñó un Esquema de Medición, Reducción y Compensación de Huella de Carbono para el sector empresarial, comercial y de servicios del DMQ, que permita a las empresas gestionar sus emisiones de gases de efecto invernadero, alimentando la meta planteada en el PMD y OT de reducción de la huella de carbono y de esta forma contribuir a la mitigación del cambio climático en el Distrito Metropolitano. Se realizaron alrededor de 4 talleres de trabajo a los que asistieron funcionarios municipales, representantes del sector privado, sociedad civil, recogiéndose importantes aportes, obteniendo al final del proceso un portafolio inicial con 4 proyectos para compensar en el sector de bosques, residuos sólidos, agricultura orgánica y sistema de bicicleta pública. (Secretaría de Ambiente Quito, 2017)

La secretaría de ambiente también tiene un proyecto vinculado a aceites y combustibles, cuyo objetivo es concientizar en el uso residencial y comercial. De igual manera para la gestión de detergentes biodegradables, propone el incentivo para la generación de convenios con productores e importadores para aumentar la presencia de detergentes biodegradables en el mercado nacional, certificaciones de producto amigable con el ambiente y el apoyo para el tratamiento de aguas residuales mediante sistemas mecánicos y químicos dentro del DMQ. (Secretaría de Ambiente Quito, 2017)

\section{Hacia una Economía Circular}

Para que las empresas implementen el modelo económico circular es de vital importancia que se impulse el modelo por medio del gobierno, ya que es necesario el apoyo y desarrollo con entidades públicas. La ciudad de Quito forma parte de 90 ciudades de todo el mundo que hacen parte del C40, red de ciudades que luchan por el cambio climático y a impulsar la acción urbana hacia la disminución de las emisiones de gases con efecto invernadero como los riesgos climáticos, y al mismo tiempo mejorar la salud, el bienestar y las oportunidades económicas para todos los ciudadanos.

El Municipio del Distrito Metropolitano de Quito fue participe del seminario de la red liderada por la ciudad de Copenhague sobre 'Crecimiento Verde', organizado en Singapur en Mayo del 2017, en donde se desarrolló un intercambio de conocimientos y lecciones aprendidas 
sobre las iniciativas de economía verde a nivel local. Ser parte del C40 es un gran paso hacia la implementación y desarrollo de este modelo económico sustentable ya que el adquirir conocimientos de la mejor manera de concebir una economía verde es a través del cambio del modelo económico actual (CONQUITO, 2017).

Alrededor del mundo existen varias empresas que han tenido gran éxito implementando el modelo económico circular, a continuación, presentamos algunos ejemplos.

Sustainer Homes: Esta compañía holandesa fabrica casas móviles totalmente desconectadas de las redes de servicios a partir de materiales reciclados y reutilizables. La calefacción y electricidad son de fuentes renovables y el agua proviene de la lluvia. Según su cofundador, las emisiones en todo el ciclo de vida representan solo un $4 \%$ de las de un hogar tradicional, sin tener que renunciar a comodidades como lavadora o lavavajillas (Evia, 2017).

Redisa: Creada para ayudar a solucionar el enorme problema de llantas que acaban en vertederos de basura de Sudáfrica, causando incendios y problemas de salud, esta compañía recolecta las llantas para reciclaje. Desde 2012 ha recogido 18 millones de ellas y creado 3 mil empleos.

New Hope Ecotech: Startup brasileña que desarrolla software para recabar y compartir datos relacionados con el reciclaje. Según la legislación del país, las empresas que ponen empaques en el mercado deben invertir en reciclaje, por lo que uno de los propósitos de New Hope Echotech es ayudar a crear cultura de la transparencia en este sentido, pero en el futuro quisiera tener una mayor influencia en el aumento del reciclaje (Evia, 2017).

Un ejemplo de empresa pionera en integrar la economía circular en su modelo de negocio es ACCIONA, que basa sus diversas actividades en la sostenibilidad. En esta línea, Acciona ha puesto en marcha: FISSAC, un proyecto europeo que desarrolla el concepto de "simbiosis industrial", para utilizar residuos de industria materia prima de otra; VETRA, para la minimización de los residuos procedentes de los procesos de limpieza de membranas en las desalinizadoras; Watintech, Aplicación De La Ósmosis Directa A La Filtración Del Agua Residual Para Aumentar Los Porcentajes De Agua Depurada O Paperchain, que propone la valorización de residuos procedentes de la industria papelera (Bermejo, 2017).

La Empresa Pública Metropolitana de Gestión Integral de Residuos Sólidos (EMGIRSEP) desarrolla varios proyectos con el objetivo de fortalecer el manejo de las más de 2.000 toneladas de basura. EMGIRS-EP empezó a disponer los residuos sólidos en un nuevo espacio dentro del Relleno Sanitario. Este sitio fue construido técnicamente en un área de 3,3 hectáreas con una profundidad de 30 metros. Durante el tercer trimestre del 2017 se opera una nueva escombrera al norte la ciudad denominada "El Semillero". Está ubicada en la comunidad de Cocotog, a 4 kilómetros del redondel de Gualo, donde se proyecta recibir un total de 1'540.236,68 metros cúbicos.

En octubre se inauguró la Planta de Generación de Energía Eléctrica a partir del Biogás que se genera de la descomposición de la basura de la ciudad y que dispone en el Relleno Sanitario. Con este proyecto se producen 5 megavatios por hora y de esta manera la capital de los 
ecuatorianos se convirtió en la ciudad pionera en el país con este tipo de Mecanismos de Desarrollo Limpio (EMGIRS-EP, 2018).

Vulgomaestre: Es la microempresa que vende camisetas con un empaque que a través de la técnica de origami se convierte en un afiche con la imagen del estampado que está en la camiseta del cliente. Una solución simple que ya incorpora conceptos y objetivos del modelo económico circular.

Cervecería Nacional: Afirma que el $90 \%$ de sus desechos recibe nuevos usos (el resto se dispone según normas ambientales). Una parte del trabajo lo hacen las plantas de Tratamiento de Aguas Residuales (PTAR), que eliminan los agentes contaminantes del agua utilizada en los procesos de producción y servicios para así obtener agua limpia. Ese líquido es reutilizado en actividades de jardinería y limpieza. A través de esta iniciativa se logró una disminución de uso de 113000 metros cúbicos de agua. Entre otras estrategias está el uso de energía térmica y plantas de generación fotovoltaica. En Quito, Cervecería Nacional cuenta con 54 paneles solares. Además, tienen un sistema de recuperación de gas metano, que se genera en las plantas de tratamiento de aguas residuales. Este ingresa a las calderas y aporta a la reducción del consumo de búnker y, a la par, de las emisiones de CO2. Con sus plantas en Guayas, Pichincha y Manabí.

Unilever: La empresa minimiza el impacto de los sachets, de alto consumo en los países con bajos ingresos. La trasnacional calcula que cada año miles de millones de estos sobrecitos de plástico son tirados a la basura. Así que optaron por aplicar tecnología para reciclaje desarrollada en Alemania, el plástico es recuperado y luego reutilizado en nuevos empaques para productos. Así se efectiviza una economía circular en acción. La empresa calcula que cada año en el mundo se pierden entre USD 80 a 120 mil millones al no reciclar adecuadamente los plásticos (Paucar, 2017)

\section{Conclusiones}

La Economía Circular supone un cambio radical de los sistemas de producción y consumo actuales. El cambio se debe dar hacia sistemas que sean regenerativos a partir de su diseño, para mantener el valor de los recursos (materiales, agua, suelo y energía) y de los productos y limitando, exponencialmente, los insumos de materias primas y energía. Esto evitará la creación de residuos e impactos negativos derivados, mitigando las externalidades negativas para el medioambiente, el clima y la salud humana.

Se pueden construir varios modelos de negocios de economía circular y trabajar con el apoyo del municipio de Quito, ya que la secretaria de ambiente en su Plan maestro de Gestión Integral de residuos plantea varios objetivos que se pueden ser cumplidos con el modelo económico circular. El cambio hacia un modelo económico circular permitirá una mejora en el manejo de residuos y se pueden crear nuevas plazas de empleo ofreciendo servicios de recolección enfocadas en el reciclaje.

El modelo de negocios circular propone un cambio no solo industrial sino también un estilo diferente de vida para las personas, mejorando la producción del sector industrial reduciendo la contaminación medio ambiental que ha existido por años, también permite la 
reutilización y reciclaje de un producto alargando su vida útil, evitando la creación de residuos e impactos ambientales.

El camino hacia una economía circular en las empresas de la ciudad de Quito apenas está en desarrollo, con la implementación de talleres a emprendedores, empresarios y microempresarios sobre esta metodología verde se podrá impulsar de mejor manera ya que se mostrarán todos los beneficios de este modelo económico y como poder aplicarlo en diferentes modalidades de negocio.

En el caso específico de Quito, a pesar de que existen políticas, ordenanzas, programas, algunos aplicados muy aisladamente, en materia de residuos sólidos urbanos, así como industriales, faltan estrategias y acciones en cada etapa del ciclo que requieren ser más eficientes en su aplicación. El proceso actual pone énfasis en la recolección, transporte y disposición, pero faltan acciones orientadas a mejorar la separación en el origen, el reciclaje, la reutilización de los residuos y trabajar en educación para la población.

La iniciativa circular en Quito reducirá la dependencia de recursos naturales, creando un valor para las empresas y los grupos de intereses, generando mayores propuestas rentables para las empresas y ayudando a minimizar el impacto ambiental que estas puedan generar.

\section{Bibliografías}

Arias, V. (2017). Un Quito Sostenible. Www.Clave.Com.Ec.

Banco Central Del Ecuador. (30 De Junio De 2017). Producto Interno Bruto. Obtenido De Https://Www.Bce.Fin.Ec/Index.Php/Boletines-De-Prensa-Archivo/Item/975-ProductoInterno-Bruto-2

Bermejo, L. (2017). Economía Circular, Un Cambio Hacia La Sostenibilidad. Http://Www.Futurosostenible.Elmundo.Es.

Bocken, N. M., Pauw, I. D., Bakker, C., \& Grinten, B. V. (2016). Product Design And Business Model Strategies For.

Canga Cabañes, J. L. (5 De Octubre De 2015). Huella De Carbono, De Agua, Ambiental De La Unión Europea. ¿Por Dónde Empiezo A Estudiar? Obtenido De Http://Www.Comunidadism.Es/Blogs/Huella-De-Carbono-De-Agua-Ambiental-De-LaUnion-Europea-\%C2\%Bfpor-Donde-Empiezo-A-Estudiar

Carra, G., \& Magdani, N. (2016). Circular Business Models for the Built Environment. Ellen Macarthur Foundation.

Conquito. (19 De Mayo De 2017). Agencia De Promoción Económica. Obtenido De Quito Entra En La Generación De Economía Circular: Http://Www.Conquito.Org.Ec/Quito-Entra-EnLa-Generacion-De-Economia-Circular/ 
Economy, 8. B. (20 De Julio De 2017). 8 Business Cases Studies for the Circular Economy. $\begin{array}{lllll}\text { Obtenido } & \text { De } & 8 & \text { Business } & \text { Cases }\end{array}$ Https://M.Box.Com/Shared_Item/Https\%3a\%2f\%2fapp.Box.Com\%2fs\%2fqaj6b57j3gnri hvek91y0otpe0xmsaoc

Ekos. (1 De Septiembre De 2015). Análisis Económico. Obtenido De Http://Www.Ekosnegocios.Com/Negocios/Verarticulocontenido.Aspx?Idart=6442

Ekos. (2 De Septiembre De 2015). La Industria En Ecuador. Obtenido De Http://Www.Ekosnegocios.Com/Negocios/Verarticulocontenido.Aspx?Idart=6442

El Comercio. (14 De Marzo De 2018). Quito Tiene 20 Contenedores Para Desechos Especiales. Obtenido De Https://Www.Elcomercio.Com/Actualidad/Contenedores-DesechosEspeciales-Emaseo-Quito.Html

Emaseo. (2017). Residuos De La Ciudad De Quito. Quito: Emaseo.

Evia, M. J. (2017). Empresas De Todo El Mundo Que Están Abrazando Los Principios De La Economía Circular. Www.Expoknews.Com/.

Jordi Morató, N. T. (2017). Situación Y Evolución De La Economía Circular. Madrid: Fundación Cotec Para La Innovación.

Jouni Korhonen, C. N. (22 De Diciembre De 2017). Science Direct. Obtenido De Circular Economy as an Essentially Contested Concept: Https://Ac.ElsCdn.Com/S0959652617330706/1-S2.0-S0959652617330706-Main.Pdf?_Tid=09937540F885-11e7-A8ca00000aacb360\&Acdnat=1515863773_A44ac265cccb1ce4c54e48ac613712d5

Korhonen, J., Honkasalo, A., \& Seppälä, J. (29 De Junio De 2017). Sciencedirect. Obtenido De Sciencedirect:

File:///C:/Users/Hpultimate/Downloads/Circular\%20economy.\%20the\%20concept\%20an $\mathrm{d} \% 20$ its\%20limitations.Pdf

Lett, L. A. (Marzo De 2014). Sciencedirect. Obtenido De Sciencedirect: Https://Ac.ElsCdn.Com/S0325754114700392/1-S2.0-S0325754114700392-Main.Pdf?_Tid=7a2cb6321684-11e8-A52f00000aacb361\&Acdnat=1519162076_8fbb5df0ba4695b54bbf302352e0c2e9

Macarthur, F. E. (2014). Towards The Circular Economy: Economic and Business Rationale for an Accelerated Transition. Ellen Macarthur Foundation.

Medioambiente, O. (2016). Observatorio.Medioambiente.Gloobal.Net. Obtenido De Http://Observatorio.Medioambiente.Gloobal.Net/Pdf/Folleto-2.Pdf 
Moreno, G. (2017). Statista.Com. Obtenido De Https://Es.Statista.Com/Grafico/9662/Los-PaisesMas-Contaminantes-Del-Mundo/

Ovacen. (S/F). Economía Circular; Qué Es Y Cómo Funciona. Obtenido De Https://Ovacen.Com/Economia-Circular/

Patricia. (14 De Febrero De 2018). El Portal De Noticias Eco-Circular De Economía Circular. Obtenido De El Portal De Noticias Eco-Circular De Economía Circular: Http://EcoCircular.Com/2018/02/14/Economia-Circular-Proyecto-De-Exito-Agua-Encaja-Mejor/

Paucar, E. (2017 De Junio De 2017). Las Empresas Aumentan Estrategias Verdes A Favor Del Ambiente. El Comercio, Págs. 1-2.

Revertia. (4 De Octubre De 2016). Pymes Y Economía Circular. Obtenido De Https://Revertia.Com/Es/Pymes-Economia-Circular/

Sandoval, V. P. (2017). Economía Circular: Relación Con La Evolución Del Concepto De. San Sebastián, España: Universidad De Navarra, Tecnun. Escuela De Ingenieros. .

Secretaria De Ambiente Alcaldía. (Junio De 2015). Contaminación Ambiental. Obtenido De Http://Aqicn.Org/Faq/2015-06-08/Wind-And-Air-Pollution-Forecast-In-Quito/

Secretaría De Ambiente Quito. (2017). Quito A Reciclar. Quito: Secretaría De Ambiente Quito. Secretaría De Ambiente Quito. (S/F). Huella De Carbono. Obtenido De Http://Huelladeciudades.Com/Calculadorasquito/Huella-Carbono.Html

Secretaria Del Medio Ambiente Quito. (2015). Políticas Y Planeación Ambiental. Obtenido De Http://Www.Quitoambiente.Gob.Ec/Ambiente/Index.Php/Politicas-Y-PlaneacionAmbiental/Residuos-Solidos/Generacion

Stahel, W. R. (2016). The Circular Economy: A New Relationship with Our Goods and Materials.

Tejedor, L. (2017). Telefónica Digitaliza La Gestión De Sus Residuos Para Impulsar La Economía Circular. Colombia: Community Manager Y Editora. Telefónica S.A. 